\title{
Checkpoint immunotherapy: good for cancer therapy, bad for rheumatic diseases
}

\author{
Leonard Calabrese, ${ }^{1}$ Vamsidhar Velcheti ${ }^{2}$
}

The goal of harnessing the immune system to fight cancer is not new; it dates back 125 years to when William Coley advocated that the body's response to infection could have anti-tumoural effects. ${ }^{1}$ However, decades of efforts using vaccines and immune stimulant therapies to harness the immune system to fight tumours have had limited success and at times have been fraught with serious adverse outcomes. Recently, drugs blocking negative immune checkpoint pathways have shown remarkable clinical activity in various solid tumours. ${ }^{2}$ Several agents targeting two such negative checkpoints, the programmed death-1 (PD-1) pathway (pembrolizumab, nivolumab and atezolizumab) and the cytotoxic T-lymphocyte-associated protein 4 (CTLA4) (ipilimumab) are currently Food and Drug Administration and European Medicines Agency approved. These negative checkpoint pathways play a role in immune tolerance in normal tissues and the activation of these pathways is largely contextual. While these therapies have added immeasurably to the longevity of many patients with malignancies, they have come at a cost with the development of numerous untoward autoimmune inflammatory conditions.

The anti-tumour mechanism for checkpoint inhibitor therapies is incompletely understood but clearly relates to the manipulation of T-cell pathways involved in cellular activation and deactivation. ${ }^{2}$ T-cell homeostasis is a tightly choreographed process whereby naïve $T$ cells rapidly proliferate in a logarithmic fashion when effectively activated and then contract following successful defeat of the challenge. CD4 T-cell activation canonically requires two signals: a primary signal (signal 1), mediated by recognition of cognate antigen by the T-cell receptor via presentation by self-major histocompatibility complex class

\footnotetext{
${ }^{1}$ Department of Rheumatology//mmunology, Cleveland Clinic, Cleveland, Ohio, USA; '2Department of Hematology and Oncology, Cleveland Clinic, Cleveland, Ohio, USA

Correspondence to Dr Leonard Calabrese,

Department of Rheumatology/Immunology, Cleveland Clinic, 9500 Euclid Drive, Cleveland, OH 44106, USA; calabrl@ccf.org
}

II molecules on the antigen-presenting cell, and a secondary signal (signal 2), mediated by CD28 on the T-cell, binding its ligand partner of the B7 family on the antigenpresenting cell. ${ }^{3}$ Of vital importance to the host is the integration of deactivating pathways or checkpoints leading to contraction of T-cell activation (figure 1). This process initially takes place in lymphoid organs and is triggered by the expression of CTLA4 on the activated T-cell. The newly expressed CTLA4 competes for B7 ligands and opposes CD28 activation, negatively regulating T-cell responses. ${ }^{3}$ In the peripheral tissues, however, PD-1 is a negative regulator of T-cell activity. The PD-1 pathway can be best understood in scenarios where activated T cells (within the context of the integrated immune response) are unable to defeat and eliminate an antigenic stimulus. This situation would be predicted to occur when the immune system is confronted by chronic replicative infections such as HIV or hepatitis $\mathrm{C}$ virus or within a tumoural environment; in such situations additional pathways are triggered to prevent collateral damage to the host from attacking T cells. ${ }^{4}$ The phenotype of such cells is often referred to as 'exhaustive' as the T-cell is still capable of effector function but is attenuated in capacity. CTLA4 also plays a role on CD8 and regulatory T cells (Treg cells): it is expressed on activated CD8 T cells where it may suppress the activity of T-helper cells downstream; in addition, it is involved in immune regulation via Treg cells as it is constitutively expressed on this cell population. ${ }^{5}$ We as rheumatologists need to appreciate that these same pathways are interdicted with the biotherapeutic agent abatacept, a fusion protein which incorporates CLTA4, and functions to inhibit signal 2. As a result of this inhibition, there is a suppressive effect on immune activation in contrast to ipilimumab which blocks CTLA4 and thereby releases an immunosuppressive checkpoint.

Attendant to the success of checkpoint inhibitor therapy are a new and wide range of toxicities of inflammatory nature with some having documented autoimmune mechanisms. There have been many recent reviews of these toxicities now referred to as immune-related adverse events
(irAEs). ${ }^{6-8}$ The most common toxicities seen with these agents include cutaneous reactions ranging from maculopapular rash to life-threatening disorders such as Sweet's syndrome, Stevens-Johnson syndrome and toxic epidermal necrolysis, among others. Gastrointestinal toxicity is common, especially diarrhoea, but a frank colitis may occur which can be lifethreatening. Endocrinopathies including thyroid disease, adrenal insufficiency and a curious syndrome of autoimmune hypophysitis are well reported. ${ }^{5-7}$ Each of these organ-based toxicities has its own incidence and chronology of onset in their irAE profiles. Finally there are a myriad of other irAEs already described involving various target organs such as the central and peripheral nervous systems, eye and pancreas. ${ }^{8}$

The fact that checkpoint therapies are associated with these irAEs should come as little surprise as there is a plethora of data leading to this expectation. The study of knockout models reveals that absence of CTLA4 is associated with a highly lethal and rapidly progressive lymphoproliferative and autoimmune disorder. ${ }^{9}$ In a spontaneous model of myasthenia gravis anti-CTLA4 has been documented to accelerate the autoimmune disease $^{10}$ and in humans anti-CTLA4 associated hypophysitis is associated with anti-pituitary antibodies believed to mediate gland destruction. ${ }^{10}$ Genetic knockouts of PD-1 produce a milder autoimmune diathesis; in addition, at least one strain of mouse develops a lupus-like diathesis when PD-1 is genetically disrupted. ${ }^{11}$ From a population health perspective, CTLA4 gene polymorphisms have been associated with an increased risk of numerous autoimmune diseases including rheumatoid arthritis. ${ }^{12}$ Additional evidence of the role of CTLA4 in control of autoimmunity stems from recent elucidation of an emerging autoimmune syndrome with multi-organ involvement associated with haploinsufficiency of CTLA4, indicating that a less than normal expression of this critical control receptor can lead to clinical immune dysregulation. ${ }^{13}$ Finally, in regard to the PD-1 pathway, recent studies have demonstrated that CD8 T-cell exhaustion, a state characterised by increased PD-1 expression, is associated with a favourable outcome in autoimmune disease and thus it could be reasoned that loss of this state could exacerbate disease progression in individuals with concomitant autoimmunity. ${ }^{14}$

In this issue of the Annals the group from Johns Hopkins elegantly documents, in the first comprehensive clinical report, 
Figure 1 Illustrations showing the T-cell activation, checkpoint inhibition and enhanced anti-tumoural response. (A) Two signal bases for effective T cell activation are shown. (B) CTLA4 mediates inhibition of T-cell activation in central lymphoid compartment and PD-1/PD-L1 or L2 mediates inhibition in peripheral tissues inducing an attenuated phenotype referred to as 'immunologic exhaustion' as these cells have reduced capacity for effector functions such as cytotoxicity and ability to promote inflammation. These nodes of immunologic control systems are referred to as checkpoints and serve to protect the host from unbridled immune activation, inflammation and autoimmunity and are attended by dampened anti-tumoural responses. (C) On inhibiting immunologic checkpoints with immune-based therapies such as anti-CTLA4 or anti-PD-1 or anti-PD-L1, reactivated $T$ cells emigrate from lymphoid compartments to seek out and engage tumours. In peripheral tissues, exhausted T cells are energised to return to a more active effector phenotype with enhanced anti-tumoural response but at a price of being more proinflammatory and capable of participating in autoimmune and auto-inflammatory reactions. CTLA4, cytotoxic T-lymphocyte-associated protein 4; MHC, major histocompatibility complex; PD-1, programmed death-1; PD-L1, programmed death ligand 1; TCR, T-cell receptor.

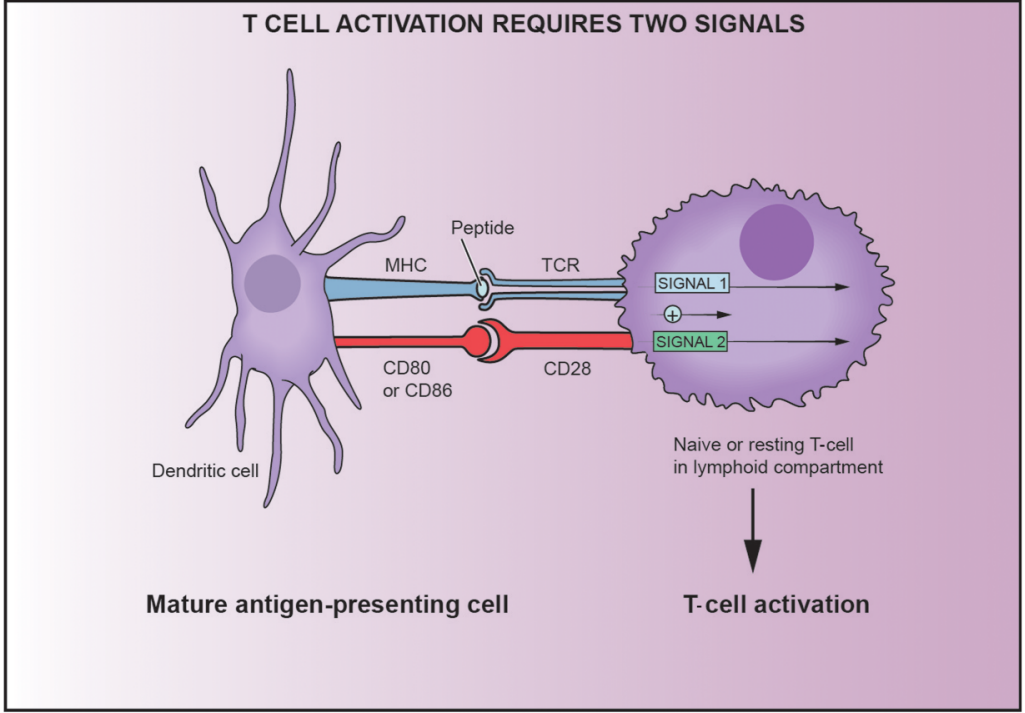

IMMUNE CHECKPOINTS CONTROL IMMUNE DEACTIVATION AND REGULATION
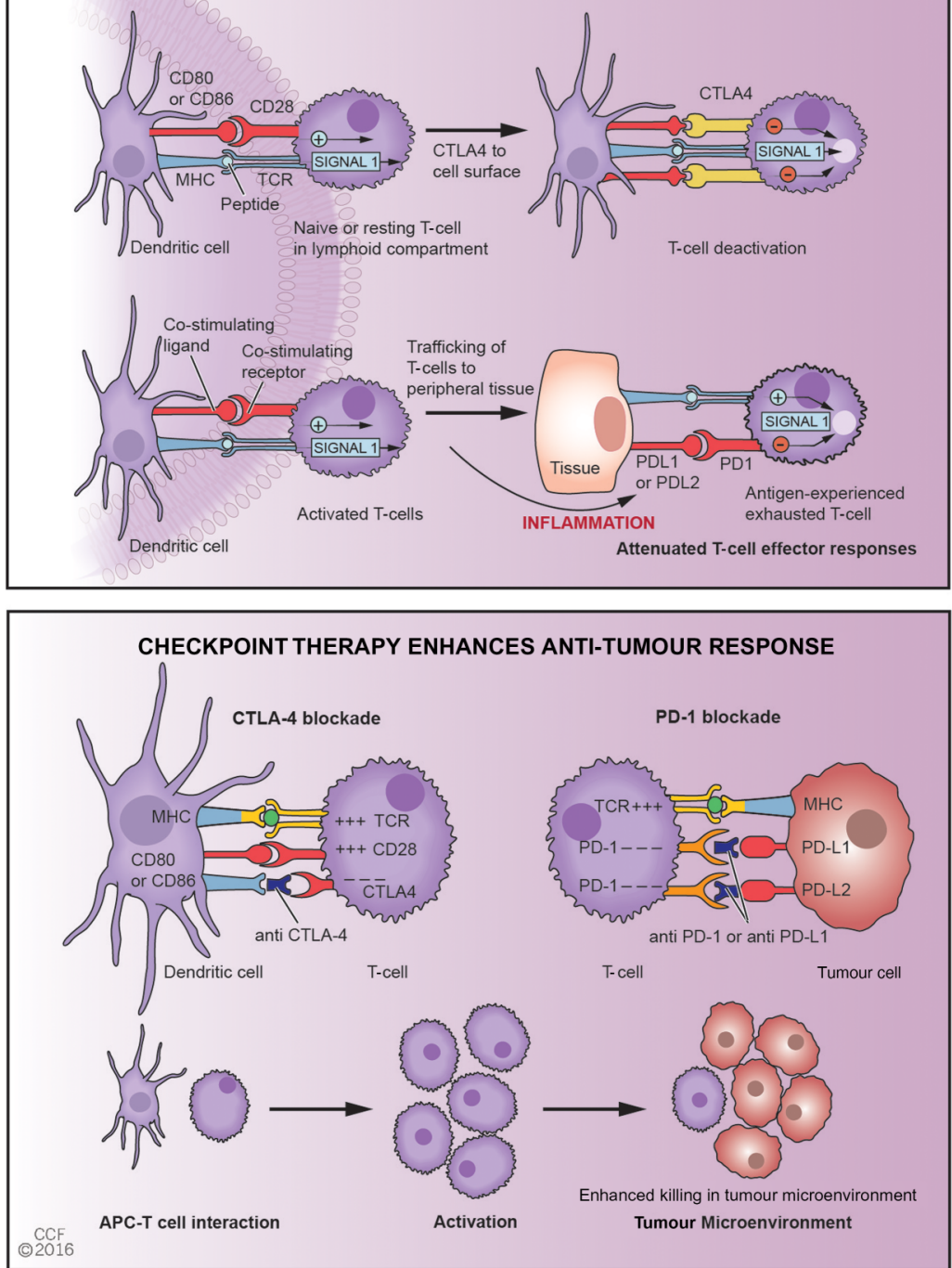

the growing spectrum of complex and at times severe rheumatic complaints occurring secondary to checkpoint therapies. ${ }^{15}$ Prior to this report, rheumatic complications have largely been described in isolated cases; no previous series has examined these irAEs in a systematic fashion. Compared with the more common irAEs, as outlined above, we have little data on the true incidence or on the relationship of such rheumatic complications with dose, duration or type of therapy (ie, antiCTLA4 vs anti-PD1 vs combination therapy) and unfortunately the design of the present study limits any insights into 
these unanswered questions. Such data are available for the more common toxicities $^{6-8}$ and the oncology community is becoming increasingly familiar with the identification and management of many of the irAEs. The very important question of whether checkpoint therapy exacerbates pre-existing autoimmunity is also poorly addressed, though limited data suggest that this phenomenon may occur in up to $40 \%$ of patients. $^{16}$ Finally, with regard to therapy, we are also only at the beginning of understanding the optimal regimens and implications of adjunctive immunosuppression, in particular as to whether the presence of irAEs may augment or hinder the anti-tumour response. One sentinel single centre study of 298 patients provides a glimpse of the magnitude and gravity of irAEs. ${ }^{17}$ In this study of lowdose anti-CTLA4 therapy for advanced melanoma, irAEs were observed in $85 \%$ of patients, with 103 patients requiring glucocorticoids and 29 patients who did not respond to initial steroid treatment requiring a second biological agent (generally an anti-tumour necrosis factor, most commonly for colitis). ${ }^{17}$

The future of checkpoint therapy is bright; there is an expanding horizon of molecular targets-some of which are already are in development-including PD-1 ligands, TIM-3, LAG-3 and others used singly and in combination. ${ }^{18}$ This also raises the spectre of new and possibly unrecognised forms of irAEs. The rheumatology community is far closer to the beginning of this entire story than some other specialties and this report should serve as a wakeup call for clinicians to familiarise themselves with these agents and the evolving literature and be poised to identify and manage such complications in concert with our oncology colleagues.

Contributors Both authors collaborated in all elements of the writing.

Competing interests None declared.

Provenance and peer review Commissioned; externally peer reviewed.

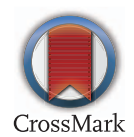

To cite Calabrese L, Velcheti V. Ann Rheum Dis 2017;76:1-3.

Received 21 June 2016

Revised 9 August 2016

Accepted 10 August 2016

Published Online First 26 August 2016

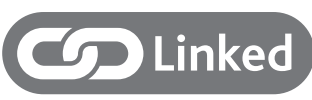

http://dx.doi.org/10.1136/annrheumdis-2016209595

Ann Rheum Dis 2017;76:1-3.

doi:10.1136/annrheumdis-2016-209782

\section{REFERENCES}

1 Brouckaert PG, Fiers W, Lejeune FJ. Coley's vaccine and TNF therapy. Nature 1992;358:630.

2 Velcheti V, Schalper K. Basic overview of current immunotherapy approaches in cancer. Am Soc Clin Oncol Educ Book 2016;35:298-308.

3 Sharma $\mathrm{P}$, Allison JP. The future of immune checkpoint therapy. Science 2015;348:56-61.

4 Blackburn SD, Shin H, Haining WN, et al. Coregulation of CD8+ T cell exhaustion by multiple inhibitory receptors during chronic viral infection. Nat Immunol 2009:10:29-37.
5 Selby MJ, Engelhardt JJ, Quigley M, et al. Anti-CTLA-4 antibodies of IgG2a isotype enhance antitumor activity through reduction of intratumoral regulatory T cells. Cancer Immunol Res 2013;1:32-42.

6 Boutros C, Tarhini A, Routier E, et al. Safety profiles of anti-CTLA-4 and anti-PD-1 antibodies alone and in combination. Nat Rev Clin Oncol 2016:13:473-86.

7 Marrone KA, Ying W, Naidoo J. Immune-related adverse events from immune checkpoint inhibitors. Clin Pharmacol Ther 2016;100:242-51.

8 Zimmer L, Goldinger SM, Hofmann L, et al. Neurological, respiratory, musculoskeletal, cardiac and ocular side-effects of anti-PD-1 therapy. Eur I Cancer 2016;60:210-25.

9 Waterhouse P, Penninger JM, Timms E, et al. Lymphoproliferative disorders with early lethality in mice deficient in Ctla-4. Science 1995;270:985-8.

10 Zumwalt TJ, Goel A. Immunotherapy of metastatic colorectal cancer: prevailing challenges and new perspectives. Curr Colorectal Cancer Rep 2015;11:125-40.

11 Nishimura H, Nose M, Hiai H, et al. Development of lupus-like autoimmune diseases by disruption of the PD-1 gene encoding an ITIM motif-carrying immunoreceptor. Immunity 1999;11:141-51.

12 Ueda H, Howson JM, Esposito L, et al. Association of the T-cell regulatory gene CTLA4 with susceptibility to autoimmune disease. Nature 2003;423:506-11.

13 Topalian SL, Sharpe AH. Balance and imbalance in the immune system: life on the edge. Immunity 2014:41:682-4.

14 McKinney EF, Lee JC, Jayne DR, et al. T-cell exhaustion, co-stimulation and clinical outcome in autoimmunity and infection. Nature 2015:523:612-16.

15 Cappelli LC, Gutierrez AK, Baer AN, et al. Inflammatory arthritis and sicca syndrome induced by nivolumab and ipilimumab. Ann Rheum Dis 2017;76:43-50.

16 Johnson DB, Sullivan RJ, Ott PA, et al. Ipilimumab therapy in patients with advanced melanoma and preexisting autoimmune disorders. JAMA Oncol 2016:2:234-40.

17 Larkin J, Hodi FS, Wolchok JD. Combined nivolumab and ipilimumab or monotherapy in untreated melanoma. N Engl J Med 2015;373:1270-1.

18 Le Mercier I, Lines JL, Noelle RJ. Beyond CTLA-4 and $\mathrm{PD}-1$, the generation $\mathrm{Z}$ of negative checkpoint regulators. Front Immunol 2015;6:418. 